\title{
ON THE CHRAPLYVY TRANSFORMATION AND SOME FEATURES OF ITS APPLICATION FOR HIGHER-ORDER EXPANSIONS
}

\author{
Alexei Turovsky \\ Bogolyubov Institute for Theoretical Physics, NAS of Ukraine, \\ Kyiv, UA-03680, Ukraine
}

(Received September 10, 2013; received in final form April 24, 2014)

\begin{abstract}
Based on Chraplyvy's generalization of the Foldy-Wouthuysen method to the two-body case, the problem of expansion of relativistic equations for two particles in powers of $1 / c$ to higher orders is considered. For the case of particles with unequal masses, the transformed Hamiltonian is obtained up to order $1 / c^{4}$. It is found that, depending on the order of application of the generating functions for the first iteration, one can get two different forms for the higher-order part of the transformed two-body Hamiltonian, which, however, can be converted one into the other by an additional unitary transformation. Ambiguities like this in the procedure are discussed. As an example for illustration, the Hermitian part of the three-dimensional Bethe-Salpeter equation with the Breit interaction is taken and its reduction to an approximate form including all the $1 / c^{4}$-order terms is carried out using the method under consideration. The results obtained here can be applied for a nonrelativistic expansion of two-body wave equations with various interaction potentials, for the study of the fine and hyperfine structure of the levels in hydrogen-like atoms. They can be of general theoretical interest as well.
\end{abstract}

Key words: Chraplyvy transformation, transformed Hamiltonian, higher orders, $1 / c$ expansion, Salpeter equation, Breit interaction.

PACS number(s): 03.65.Pm, 03.65.Ca, 02.30.Mv, 31.30.jy

\section{INTRODUCTION}

In spite of considerable achievements in quantum field theory, particularly in quantum electrodynamics (QED), relativistic two-body wave equations are widely used to learn about various relativistic effects in quantum systems and also to study and calculate their energy spectra (see, e.g., [1-6] and references therein). However, to get an acceptable description of a two-body system, it is not essential to solve the original equations of motion, and one can often restrict oneself to the consideration of their approximate forms with a small parameter, such as the inverse speed of light. As a rule, for many applications, they usually restrict themselves to an expansion of wave equations in powers of $1 / c$ to second order. Thus, e.g., the well-known Breit-Fermi Hamiltonian and its special case for a vector exchange, which can be derived from the nonrelativistic reduction of different two-body equations, are often used to describe spectra of atomic, nuclear, and quark systems [7-9].

A relatively simple description of a system of two spinhalf particles, including relativistic effects and its energy spectrum, can be given with the help of equations of the Breit type. In order to obtain a semirelativistic approximation of equations of this type, they usually apply the Foldy-Wouthuysen (FW) method [10] generalized by Chraplyvy to the two-body problem $[11,12]$.

According to this method, one represents a relativistic two-body Hamiltonian in the following general form:

$$
H=\beta_{1} m_{1} c^{2}+\beta_{2} m_{2} c^{2}+(\mathcal{E} \mathcal{E})+(\mathcal{E O})+(\mathcal{O E})+(\mathcal{O O})
$$

This is a sum of the two "large" terms $\beta_{1} m_{1} c^{2}+\beta_{2} m_{2} c^{2}$ and even-even, even-odd, odd-even, and odd-odd terms, respectively. In the case of two Dirac particles, they are, in fact, matrices of $16 \times 16=256$ elements, and can be written as direct products of four-by-four matrices of each particle. In order to remove the undesirable terms (even-odd, etc.), the Hamiltonian must be subjected to canonical transformations of the type

$$
\begin{aligned}
e^{i S} H e^{-i S} & =H+i[S, H]+\frac{(i)^{2}}{2 !}[S,[S, H]] \\
& +\frac{(i)^{3}}{3 !}[S,[S,[S, H]]]+\cdots,
\end{aligned}
$$

provided that the generating functions $S$ are suitablychosen Hermitian operators, and where the inverse speed of light (or, equivalently, the inverse masses) can be taken as an expansion parameter.

Though in the case of interacting particles one cannot determine a unitary transformation which cancels all the undesirable terms in all orders in principle, the original Hamiltonian can be converted by the procedure (2) into an even-even operator to any desired degree of approximation. There are a lot of sets of $S$ to reduce a two-body Hamiltonian to approximate forms $[12,13]$. In this paper, we apply the generating functions in a simple form, which, for the first iteration, read as follows:

$$
\begin{aligned}
S_{o e} & =-\frac{i \beta_{1}}{2 m_{1} c^{2}}(\mathcal{O} \mathcal{E}), \\
S_{e o} & =-\frac{i \beta_{2}}{2 m_{2} c^{2}}(\mathcal{E} \mathcal{O}), \\
S_{\text {oo }} & =-\frac{i\left(\beta_{1} m_{1}-\beta_{2} m_{2}\right)}{2\left(m_{1}^{2}-m_{2}^{2}\right) c^{2}}(\mathcal{O O}) .
\end{aligned}
$$


The generators for the next iterations differ from (3) by the structure of their odd-even, even-odd, and odd-odd factors and enable us to reduce a sixteen-component twobody equation to its four-component approximate forms relative to the chosen energy states of the particles. They were first introduced by Chraplyvy in [11] to convert the two-body Hamiltonian (1) into an even-even operator to order $1 / c^{2}$ (under the assumption that $(\mathcal{E} \mathcal{E}),(\mathcal{O O})$ are of order $c^{0}$, and $(\mathcal{O E}),(\mathcal{E O})$ of order $\left.c^{1}\right)$,

$$
\begin{aligned}
& H_{\mathrm{tr}}=\beta_{1} m_{1} c^{2}+\beta_{2} m_{2} c^{2}+(\mathcal{E} \mathcal{E}) \\
& +\frac{\beta_{1}}{2 m_{1} c^{2}}(\mathcal{O} \mathcal{E})^{2}+\frac{\beta_{2}}{2 m_{2} c^{2}}(\mathcal{E} \mathcal{O})^{2} \\
& +\frac{1}{8 m_{1}^{2} c^{4}}[(\mathcal{O} \mathcal{E}),[(\mathcal{E} \mathcal{E}),(\mathcal{O} \mathcal{E})]] \\
& +\frac{1}{8 m_{2}^{2} c^{4}}[(\mathcal{E O}),[(\mathcal{E} \mathcal{E}),(\mathcal{E O})]] \\
& -\frac{\beta_{1}}{8 m_{1}^{3} c^{6}}(\mathcal{O} \mathcal{E})^{4}-\frac{\beta_{2}}{8 m_{2}^{3} c^{6}}(\mathcal{E O})^{4} \\
& +\frac{\beta_{1} \beta_{2}}{8 m_{1} m_{2} c^{4}}\left\{\left[(\mathcal{O E}),[(\mathcal{E O}),(\mathcal{O O})]_{+}\right]_{+}\right. \\
& \left.+\left[(\mathcal{E O}),[(\mathcal{O E}),(\mathcal{O O})]_{+}\right]_{+}\right\} \\
& +\frac{\beta_{1} m_{1}-\beta_{2} m_{2}}{2\left(m_{1}^{2}-m_{2}^{2}\right) c^{2}}(\mathcal{O O})^{2} \\
& +\frac{\beta_{2} m_{1}-\beta_{1} m_{2}}{8 m_{1} m_{2}\left(m_{1}^{2}-m_{2}^{2}\right) c^{6}}[(\mathcal{O E}),(\mathcal{E O})]^{2} \\
& -\frac{\beta_{1} m_{1}+\beta_{2} m_{2}}{16 m_{1}^{2} m_{2}^{2} c^{6}}\left[(\mathcal{O E})^{2},(\mathcal{E O})^{2}\right]_{+} \\
& +\frac{\beta_{1}}{8 m_{1} m_{2}^{2} c^{6}}(\mathcal{E O})(\mathcal{O} \mathcal{E})^{2}(\mathcal{E O}) \\
& +\frac{\beta_{2}}{8 m_{1}^{2} m_{2} c^{6}}(\mathcal{O E})(\mathcal{E O})^{2}(\mathcal{O E}) \\
& +\frac{\beta_{1} \beta_{2}\left(m_{1}^{2}+m_{2}^{2}\right)-2 m_{1} m_{2}}{8 m_{1} m_{2}\left(m_{1}^{2}-m_{2}^{2}\right) c^{4}} \\
& \times[[(\mathcal{E O}),(\mathcal{O E})],(\mathcal{O O})] .
\end{aligned}
$$

Because of the form of $(3 \mathrm{c})$ this prescription is applicable for the case of unequal masses only, however, it enables one to get four forms of the reduced Hamiltonian relative to the four different energy states of the system.

They often use prescription (4) in atomic physics. For example, it was applied by Barker and Glover in order to transform the Salpeter equation [14], which they hermitize for this purpose, and the Breit equation with the Breit interaction and a phenomenological correction taking into account the intrinsic magnetic moments of the particles to sixteen-component approximate forms [15]. Using the obtained transformed Hamiltonians, correct to $1 / c^{2}$, they carried out a perturbation calculation of the fine and hyperfine structure for hydrogen and positronium up to the order $\alpha^{5} m c^{2}$.

Although, as a rule, the Chraplyvy transformation is often used to order $1 / c^{2}$, so that they restrict themselves to Eq. (4), the properties and features of the transformed Hamiltonians valid to the $n$th order in expansion parameters are discussed in the literature $[16,17]$. Still, the twobody, and $n$-body in general, transformation to higher orders in $1 / c$ can be of some interest not only from the theoretical point of view. Thus, e.g., in addition to the effects of the order $1 / c^{2}$, relativistic equations for Dirac particles with electromagnetic interactions contain some information about the form of terms giving relativistic and radiative corrections up to $1 / c^{3}$ and $1 / c^{4}$, and which can also be obtained from the nonrelativistic reduction $[18,19]$. However, in a general case, one needs to apply QED to get the $\alpha^{5} m c^{2}, \alpha^{6} m c^{2}$ corrections to the energy (see, e.g., [20-26]), but the problem of derivation of the higher-order effective Hamiltonian for an arbitrary light atom remains difficult. Nevertheless, an expansion of relativistic equations to higher orders provides a rather straightforward derivation of an effective Hamiltonian for bound states, although this way does not give a complete treatment of relativistic and quantum field effects.

This paper focuses on some further development of the two-body reduction techniques of Chraplyvy and also its application to the problem of the expansion of equaltime relativistic wave equations for two Dirac particles up to the order $1 / c^{4}$, with the help of the generating functions (3), and it is organized as follows. Section II deals with the two-body Hamiltonian transformed to higher orders, for which all the $1 / c^{4}$-order terms are found, and which is a continuation of the transformation (4) for the case of commutation of $(\mathcal{O E})$ and $(\mathcal{E O})$. It occurs that the form of the higher-order part of the transformed Hamiltonian depends on the order of application of the functions (3), namely, it can involve certain extra terms having a difference of masses in the denominators even though both particles are in a positive energy state. Here, we are concerned with additional unitary transformations canceling terms of this type as well.

In Section III, for the sake of illustration, we consider the $1 / c$ expansion of the Salpeter equation (its Hermitian part, to be exact) with the Breit interaction, and calculate all the terms of the order $1 / c^{4}$ in its transformed Hamiltonian. Finally, Section IV contains a summary and conclusions of the article.

\section{THE TRANSFORMED HAMILTONIAN}

Proceeding with the procedure of transformation of the Hamiltonian (1), using generators (3), and with due regard for the commutation relation

$$
[(\mathcal{O} \mathcal{E}),(\mathcal{E} \mathcal{O})]=0
$$

which causes a considerable simplification in $H_{\mathrm{tr}}$, we get new even-even terms coming after the terms written in Eq. (4), and which are of a lower order of magnitude. They form the higher-order, with respect to $1 / c$, part of the transformed Hamiltonian.

Thus one obtains the following prescription for the transformation of $H$ : 


$$
\begin{aligned}
& H_{\mathrm{tr}}=\beta_{1} m_{1} c^{2}+\beta_{2} m_{2} c^{2}+(\mathcal{E} \mathcal{E})+\frac{\beta_{1}}{2 m_{1} c^{2}}(\mathcal{O E})^{2}+\frac{\beta_{2}}{2 m_{2} c^{2}}(\mathcal{E O})^{2}+\frac{\beta_{1} m_{1}-\beta_{2} m_{2}}{2\left(m_{1}^{2}-m_{2}^{2}\right) c^{2}}(\mathcal{O O})^{2} \\
& +\frac{1}{8 m_{1}^{2} c^{4}}[(\mathcal{O E}),[(\mathcal{E} \mathcal{E}),(\mathcal{O E})]]+\frac{1}{8 m_{2}^{2} c^{4}}[(\mathcal{E O}),[(\mathcal{E} \mathcal{E}),(\mathcal{E O})]]+\frac{\beta_{1} \beta_{2}}{4 m_{1} m_{2} c^{4}}\left[(\mathcal{O E}),[(\mathcal{E O}),(\mathcal{O O})]_{+}\right]_{+} \\
& -\frac{\beta_{1}}{8 m_{1}^{3} c^{6}}(\mathcal{O E})^{4}-\frac{\beta_{2}}{8 m_{2}^{3} c^{6}}(\mathcal{E O})^{4} \\
& -\frac{\beta_{1}}{8 m_{1}^{3} c^{6}}[(\mathcal{O E}),(\mathcal{E} \mathcal{E})]^{2}-\frac{\beta_{2}}{8 m_{2}^{3} c^{6}}[(\mathcal{E O}),(\mathcal{E} \mathcal{E})]^{2} \\
& +\frac{\beta_{1}}{8 m_{1} m_{2}^{2} c^{6}}[(\mathcal{E O}),(\mathcal{O O})]_{+}^{2}+\frac{\beta_{2}}{8 m_{1}^{2} m_{2} c^{6}}[(\mathcal{O E}),(\mathcal{O O})]_{+}^{2} \\
& -\frac{\beta_{1} m_{1}-\beta_{2} m_{2}}{16 m_{1}^{2}\left(m_{1}^{2}-m_{2}^{2}\right) c^{6}}\left[(\mathcal{O O}),\left[(\mathcal{O E}),[(\mathcal{O E}),(\mathcal{O O})]_{+}\right]_{+}\right]_{+}-\frac{\beta_{1}\left(\beta_{1} m_{1}-\beta_{2} m_{2}\right)^{2}}{16 m_{1}\left(m_{1}^{2}-m_{2}^{2}\right)^{2} c^{6}}\left[(\mathcal{O O}),\left[(\mathcal{O O}),(\mathcal{O E})^{2}\right]_{+}\right]_{+} \\
& -\frac{\beta_{1} m_{1}-\beta_{2} m_{2}}{16 m_{2}^{2}\left(m_{1}^{2}-m_{2}^{2}\right) c^{6}}\left[(\mathcal{O O}),\left[(\mathcal{E O}),[(\mathcal{E O}),(\mathcal{O O})]_{+}\right]_{+}\right]_{+}-\frac{\beta_{2}\left(\beta_{1} m_{1}-\beta_{2} m_{2}\right)^{2}}{16 m_{2}\left(m_{1}^{2}-m_{2}^{2}\right)^{2} c^{6}}\left[(\mathcal{O O}),\left[(\mathcal{O O}),(\mathcal{E O})^{2}\right]_{+}\right]_{+} \\
& -\frac{\beta_{1} m_{2}-\beta_{2} m_{1}}{8 m_{1} m_{2}\left(m_{1}^{2}-m_{2}^{2}\right) c^{6}}[(\mathcal{O O}),[(\mathcal{O E}),[(\mathcal{E O}),(\mathcal{E} \mathcal{E})]]]_{+} \\
& \left.+\frac{\beta_{1}}{8 m_{1} m_{2}^{2} c^{6}}[(\mathcal{E O}),(\mathcal{E} \mathcal{E})],[(\mathcal{O E}),(\mathcal{O O})]_{+}\right]+\frac{\beta_{2}}{8 m_{1}^{2} m_{2} c^{6}}\left[[(\mathcal{O E}),(\mathcal{E E})],[(\mathcal{E O}),(\mathcal{O O})]_{+}\right] \\
& +\frac{\left(\beta_{1} m_{1}-\beta_{2} m_{2}\right)^{2}}{8\left(m_{1}^{2}-m_{2}^{2}\right)^{2} c^{4}}[(\mathcal{O O}),[(\mathcal{E E}),(\mathcal{O O})]] \\
& +\frac{1}{384 m_{1}^{4} c^{8}}\left\{[(\mathcal{O E}),[(\mathcal{O E}),[(\mathcal{O E}),[(\mathcal{O E}),(\mathcal{E E})]]]]+32\left[(\mathcal{O E})^{3},[(\mathcal{O} \mathcal{E}),(\mathcal{E} \mathcal{E})]\right]\right\} \\
& +\frac{1}{384 m_{2}^{4} c^{8}}\left\{[(\mathcal{E O}),[(\mathcal{E O}),[(\mathcal{E O}),[(\mathcal{E O}),(\mathcal{E E})]]]]+32\left[(\mathcal{E O})^{3},[(\mathcal{E O}),(\mathcal{E} \mathcal{E})]\right]\right\} \\
& +\frac{1}{64 m_{1}^{2} m_{2}^{2} c^{8}}[(\mathcal{O} \mathcal{E}),[(\mathcal{O E}),[(\mathcal{E O}),[(\mathcal{E O}),(\mathcal{E} \mathcal{E})]]]] \\
& -\frac{\beta_{1} \beta_{2}}{96 m_{1}^{3} m_{2} c^{8}}\left\{\left[(\mathcal{O E}),\left[(\mathcal{O E}),\left[(\mathcal{O E}),[(\mathcal{E O}),(\mathcal{O O})]_{+}\right]_{+}\right]_{+}\right]_{+}+8\left[(\mathcal{O E})^{3},[(\mathcal{E O}),(\mathcal{O O})]_{+}\right]_{+}\right\} \\
& -\frac{\beta_{1} \beta_{2}}{96 m_{1} m_{2}^{3} c^{8}}\left\{\left[(\mathcal{O} \mathcal{E}),\left[(\mathcal{E O}),\left[(\mathcal{E O}),[(\mathcal{E O}),(\mathcal{O O})]_{+}\right]_{+}\right]_{+}\right]_{+}+8\left[(\mathcal{O E}),\left[(\mathcal{E O})^{3},(\mathcal{O O})\right]_{+}\right]_{+}\right\} \\
& +\frac{\beta_{1}}{16 m_{1}^{5} c^{10}}(\mathcal{O E})^{6}+\frac{\beta_{2}}{16 m_{2}^{5} c^{10}}(\mathcal{E O})^{6} .
\end{aligned}
$$

The FW method allows one to expand the Hamiltonian to any desired degree of approximation, keeping its Hermitian character. Here, we assume that $(\mathcal{E E}),(\mathcal{O O})$ are of the order $c^{0}$, and $(\mathcal{O E}),(\mathcal{E O})$ of the order $c^{1}$, and we retain the terms up to order $1 / c^{4}$. So, under this assumption, expression (6) represents the transformed Hamiltonian approximate out to the fourth order. It can be divided into two parts. The first part consists of the terms (6a) and the transformed Hamiltonian is correct to $1 / c^{2}$, into which expression (4) goes over under the commutation relation (5). The second part, which we will refer to as the higher-order transformed Hamilto- nian, involves all the $1 / c^{4}$-order terms $(6 \mathrm{~b} \ldots \mathrm{j})$. It is a sixteen-component equation, and as usual, to obtain its four-component forms, i.e. reduced Hamiltonians, one has to put $\beta_{1}=\beta_{2}= \pm 1$ or $\beta_{1}=-\beta_{2}= \pm 1$. Notice that if the inverse mass is considered as an expansion parameter, the terms $(6 \mathrm{~b} \ldots$ e), which are nonlinear in $(\mathcal{E E})$ and $(\mathcal{O O})$, are of the order $1 / \mathrm{m}^{3}$. Many of them have a mass difference in the denominators. The terms $(6 \mathrm{~g}, \mathrm{~h}$, i), which are linear in $(\mathcal{E E}),(\mathcal{O O})$, are of the order $1 / \mathrm{m}^{4}$. The term (6f), which consists of the even-even and oddodd operators, is the only one of the order $1 / \mathrm{m}^{2}$ in the higher-order transformed Hamiltonian. 


\section{ALEXEI TUROVSKY}

For the case when the mass of one particle becomes considerably great in comparion with the mass of the other one, i.e. $m_{1} \rightarrow \infty$ or $m_{2} \rightarrow \infty$, equation (6) goes over into the corresponding formula for a single Dirac particle in external fields:

$$
\begin{aligned}
H_{\mathrm{tr}} & =\beta m c^{2}+\mathcal{E}+\frac{\beta}{2 m c^{2}} \mathcal{O}^{2}+\frac{1}{8 m^{2} c^{4}}[\mathcal{O},[\mathcal{E}, \mathcal{O}]] \\
& -\frac{\beta}{8 m^{3} c^{6}} \mathcal{O}^{4}-\frac{\beta}{8 m^{3} c^{6}}[\mathcal{O}, \mathcal{E}]^{2} \\
& +\frac{1}{384 m^{4} c^{8}}[\mathcal{O},[\mathcal{O},[\mathcal{O},[\mathcal{O}, \mathcal{E}]]]] \\
& +\frac{1}{12 m^{4} c^{8}}\left[\mathcal{O}^{3},[\mathcal{O}, \mathcal{E}]\right]+\frac{\beta}{16 m^{5} c^{10}} \mathcal{O}^{6}
\end{aligned}
$$

which is the transformation of the Hamiltonian

$$
H=\beta m c^{2}+\mathcal{E}+\mathcal{O},
$$

with the use of the generator

$$
S=-\frac{i \beta}{2 m c^{2}} \mathcal{O}
$$

Under this condition only the terms of $(6 \mathrm{~b}, \mathrm{~g}, \mathrm{j})$ remain in the higher-order transformed Hamiltonian and pass into the last four terms in Eq. (7); the rest of the commutators and anticommutators, which involve the $(\mathcal{O O})$ terms in expression (6), vanish.

The transformed Hamiltonian written out in Eq. (4) to the second order is derived with the use of the generating functions (3), and it is not so important which of them is used first in the first iteration in series (2); regardless of the order of their application in this iteration, the same expression in the form of (4) will be obtained. In other words, it is not important which of the undesirable terms in Eq. (1) will be destroyed first. But this statement is correct only if one needs to get the expansion up to the terms written in Eq. (4), or the same, to the second order in $1 / c$ under our assumption. The use of $S_{o e}$ or $S_{e o}$ first in the sequence in the first iteration leads to the transformed Hamiltonian in the form (6), however, if one applies $S_{o o}$ first (instead of $S_{o e}$ or $S_{e o}$ ) in (2), certain "extra" terms of the fourth order will arise in the transformed Hamiltonian in addition to those in Eq. (6); namely,

$$
\begin{aligned}
& \frac{\beta_{1} m_{2}-\beta_{2} m_{1}}{8 m_{1} m_{2}\left(m_{1}^{2}-m_{2}^{2}\right) c^{6}}[[(\mathcal{O} \mathcal{E})(\mathcal{E O}),(\mathcal{O O})],(\mathcal{E} \mathcal{E})] \\
& +\frac{\beta_{1} m_{2}+\beta_{2} m_{1}}{8 m_{1} m_{2}\left(m_{1}^{2}-m_{2}^{2}\right) c^{6}} \\
& \times[(\mathcal{E} \mathcal{O})(\mathcal{O O})(\mathcal{O E})-(\mathcal{O E})(\mathcal{O O})(\mathcal{E O}),(\mathcal{E} \mathcal{E})] \\
& +\frac{m_{2}-\beta_{1} \beta_{2} m_{1}}{16 m_{1}^{2} m_{2}\left(m_{1}^{2}-m_{2}^{2}\right) c^{8}}\left[[(\mathcal{O E})(\mathcal{E O}),(\mathcal{O O})],(\mathcal{O E})^{2}\right] \\
& +\frac{\beta_{1} \beta_{2} m_{2}-m_{1}}{16 m_{1} m_{2}^{2}\left(m_{1}^{2}-m_{2}^{2}\right) c^{8}}\left[[(\mathcal{O E})(\mathcal{E O}),(\mathcal{O O})],(\mathcal{E O})^{2}\right] \\
& +\frac{m_{2}+\beta_{1} \beta_{2} m_{1}}{16 m_{1}^{2} m_{2}\left(m_{1}^{2}-m_{2}^{2}\right) c^{8}} \\
& \times\left[(\mathcal{E O})(\mathcal{O O})(\mathcal{O E})-(\mathcal{O E})(\mathcal{O O})(\mathcal{E O}),(\mathcal{O E})^{2}\right] \\
& +\frac{\beta_{1} \beta_{2} m_{2}+m_{1}}{16 m_{1} m_{2}^{2}\left(m_{1}^{2}-m_{2}^{2}\right) c^{8}} \\
& \times\left[(\mathcal{E O})(\mathcal{O O})(\mathcal{O E})-(\mathcal{O E})(\mathcal{O O})(\mathcal{E O}),(\mathcal{E O})^{2}\right] .
\end{aligned}
$$

As follows from this equation, the terms having a mass difference in the denominators appear even though both particles are in positive or negative energy states (those correspond to setting $\beta_{1}=\beta_{2}= \pm 1$ ). Such terms, but with other numerical factors, also appear in $H_{\mathrm{tr}}$, if one takes the sum $S=S_{o e}+S_{e o}+S_{o o}$ as a generator [27]. Obviously, some extra terms enter the expansion in the sixth order and higher. The dependence of the form for a higher-order part of the transformed Hamiltonian on the order of application of the generating functions can be an important feature of the expansion to higher orders, and makes the difference between the second-order and higher-order expansions.

Nevertheless, terms (8) can be eliminated provided that the transformed Hamiltonian is subjected to an additional unitary transformation like (2) with a generating function in the form of a Hermitian even-even operator, which we represent in such a manner:

$$
\begin{aligned}
S_{e e} & =-\frac{i\left(\beta_{1} m_{2}-\beta_{2} m_{1}\right)}{8 m_{1} m_{2}\left(m_{1}^{2}-m_{2}^{2}\right) c^{6}}[(\mathcal{O O}),(\mathcal{O E})(\mathcal{E O})] \\
& -\frac{i\left(\beta_{1} m_{2}+\beta_{2} m_{1}\right)}{8 m_{1} m_{2}\left(m_{1}^{2}-m_{2}^{2}\right) c^{6}} \\
& \times\{(\mathcal{O} \mathcal{E})(\mathcal{O O})(\mathcal{E O})-(\mathcal{E O})(\mathcal{O O})(\mathcal{O} \mathcal{E})\}
\end{aligned}
$$

Taking into account its higher order, one can retain only the first two terms in series (2), while the rest of the terms might be discarded because they are of a lower order of magnitude,

$$
e^{i S_{e e}} H_{\mathrm{tr}} e^{-i S_{e e}} \approx H_{\mathrm{tr}}+i\left[S_{e e}, H_{\mathrm{tr}}\right]
$$

Since $S_{e e}$ has an even-even form, it commutes with the two large terms from $H_{\mathrm{tr}}$ :

$$
\left[S_{e e}, \beta_{1} m_{1} c^{2}+\beta_{2} m_{2} c^{2}\right]=0
$$

Actually, to remove the terms (8), it is quite convenient to retain the members of the order $c^{0}$ in $H_{\text {tr }}$ standing in the commutator in (10) and to omit the rest. It is sufficient to get the terms which coincide with the ones in Eq. (8) up to a sign. One sees that the operators (9a) and (9b) act separately. The former destroys the first, third, and fourth terms in Eq. (8), and the latter destroys the rest. We note that in general while removing the extra terms, this procedure gives rise to the new ones instead, but all of them are of a lower order of magnitude.

Thus the generating function $S_{e e}$ allows one to modify the higher-order transformed Hamiltonian, subtracting (or removing) the terms (8). One can express it in terms of the original operators $S_{o e}, S_{e o}$, and $S_{o o}$ in a convenient brief form:

$$
S_{e e}=\left[S_{o e},\left[S_{e o}, S_{o o}\right]\right] .
$$

Still, this additional transformation is not the only one, and any transformation with Hermitian even-even functions can be used to modify the higher-order transformed 
Hamiltonian. Using transformations of this kind, one can destroy and create a number of higher-order terms in the approximate Hamiltonian (see, e.g., [28]). Meanwhile, as a rule, these procedures, except the one considered above, produce new terms instead of the removed ones.

In conclusion of this section we should point out that if the commutation relation (5) had not been satisfied, the expression for $H_{\mathrm{tr}}$ would be much lengthier than that in Eq. (6) and many new terms would appear in it as well. Moreover, the structure of the extra terms and thus of the generator $S_{e e}$ would be more complicated than that we had in equations (8), (9a), (9b) and (12).

\section{APPLICATION TO THE SALPETER EQUATION}

Consider the Hamiltonian of the Hermitian part of the three-dimensional Bethe-Salpeter equation in coordinate space $[14,15]$ :

$$
H=H_{1}+H_{2}+\frac{1}{2}\left[\left(\Lambda_{1}^{+} \Lambda_{2}^{+}-\Lambda_{1}^{-} \Lambda_{2}^{-}\right), V(\mathbf{r})\right]_{+},
$$

where

$$
\begin{array}{cl}
H_{1}=c \boldsymbol{\alpha}_{1} \cdot \mathbf{p}_{1}+\beta_{1} m_{1} c^{2}, & H_{2}=c \boldsymbol{\alpha}_{2} \cdot \mathbf{p}_{2}+\beta_{2} m_{2} c^{2}, \\
\Lambda_{1}^{ \pm}=\frac{E_{1} \pm H_{1}}{2 E_{1}}, & \Lambda_{2}^{ \pm}=\frac{E_{2} \pm H_{2}}{2 E_{2}}, \\
E_{1}=+\left(m_{1}^{2} c^{4}+\mathbf{p}_{1}^{2} c^{2}\right)^{1 / 2}, & E_{2}=+\left(m_{2}^{2} c^{4}+\mathbf{p}_{2}^{2} c^{2}\right)^{1 / 2} .
\end{array}
$$

Our main goal here is to apply the results obtained above to get the transformation of Hamiltonian (13) up to order $1 / c^{4}$. For illustrative purposes, we will consider the case of particles with unequal masses and of charges $\epsilon_{1}$ and $\epsilon_{2}$, interacting through the potential

$$
V(\mathbf{r})=\frac{\epsilon_{1} \epsilon_{2}}{r}-\frac{\epsilon_{1} \epsilon_{2}}{2 r}\left(\boldsymbol{\alpha}_{1} \cdot \boldsymbol{\alpha}_{2}+\frac{\left(\boldsymbol{\alpha}_{1} \cdot \mathbf{r}\right)\left(\boldsymbol{\alpha}_{2} \cdot \mathbf{r}\right)}{r^{2}}\right),
$$

where $\mathbf{r}=\mathbf{r}_{1}-\mathbf{r}_{2}$ and $r=|\mathbf{r}|$. One should remember, however, that in general the complete form of the original interaction of two electromagnetic particles also has to include many other components of lower order of magnitude in addition to the Breit interaction (14), e.g., such as the intrinsic magnetic moment terms [15], the terms involving the electron self-energy and vacuum polarization [29], etc. Nevertheless, here for simplicity we restrict ourselves to consideration of an expansion of the equation only with the potential in the form (14).

Using the expression (6a), one performs the $1 / c$ expansion of the Hamiltonian (13) to the second order and, putting $\beta_{1}=\beta_{2}=1$, gets the Breit correction derived also in QED (see, e.g., [30,31]). Based on Eq. (4), or the same on part (6a), the expansion of (13) to order $1 / c^{2}$ and the study of the properties of the obtained transformed Hamiltonian were carried out by Barker and
Clover [15]. Note that the Breit correction is divergent as it involves the Dirac $\delta$-functions appearing because of the Coulomb singularity in the initial interaction, and because of the same singularity, the $\delta$-functions, already together with their derivatives, will appear as well and in the expansion terms in higher orders.

In order to reduce the Hamiltonian to form (1), we evaluate the anticommutator, keeping the terms which contribute up to $1 / c^{4}$ in the expansion. Hence we have

$$
\begin{aligned}
& (\mathcal{E} \mathcal{E})=\left(\beta_{1}+\beta_{2}\right) \frac{\epsilon_{1} \epsilon_{2}}{2 r}-\left[\frac{\beta_{1} \mathbf{p}_{1}^{2}}{8 m_{1}^{2} c^{2}}+\frac{\beta_{2} \mathbf{p}_{2}^{2}}{8 m_{2}^{2} c^{2}}, \frac{\epsilon_{1} \epsilon_{2}}{r}\right]_{+} \\
& +\left[\frac{3 \beta_{1} \mathbf{p}_{1}^{4}}{32 m_{1}^{4} c^{4}}+\frac{3 \beta_{2} \mathbf{p}_{2}^{4}}{32 m_{2}^{4} c^{4}}, \frac{\epsilon_{1} \epsilon_{2}}{r}\right]_{+}, \\
& (\mathcal{O E})=c \boldsymbol{\alpha}_{1} \cdot \mathbf{p}_{1}+\left[\frac{\boldsymbol{\alpha}_{1} \cdot \mathbf{p}_{1}}{4 m_{1} c}-\frac{\left(\boldsymbol{\alpha}_{1} \cdot \mathbf{p}_{1}\right) \mathbf{p}_{1}^{2}}{8 m_{1}^{3} c^{3}}, \frac{\epsilon_{1} \epsilon_{2}}{r}\right]_{+} \\
& -\left[\frac{\boldsymbol{\alpha}_{2} \cdot \mathbf{p}_{2}}{4 m_{2} c}, \frac{\epsilon_{1} \epsilon_{2}}{2 r}\left(\delta^{i j}+\frac{r^{i} r^{j}}{r^{2}}\right) \alpha_{1}^{i} \alpha_{2}^{j}\right]_{+} \\
& +\left[\frac{\left(\boldsymbol{\alpha}_{2} \cdot \mathbf{p}_{2}\right) \mathbf{p}_{2}^{2}}{8 m_{2}^{3} c^{3}}, \frac{\epsilon_{1} \epsilon_{2}}{2 r}\left(\delta^{i j}+\frac{r^{i} r^{j}}{r^{2}}\right) \alpha_{1}^{i} \alpha_{2}^{j}\right]_{+}, \\
& (\mathcal{E} \mathcal{O})=c \boldsymbol{\alpha}_{2} \cdot \mathbf{p}_{2}+\left[\frac{\boldsymbol{\alpha}_{2} \cdot \mathbf{p}_{2}}{4 m_{2} c}-\frac{\left(\boldsymbol{\alpha}_{2} \cdot \mathbf{p}_{2}\right) \mathbf{p}_{2}^{2}}{8 m_{2}^{3} c^{3}}, \frac{\epsilon_{1} \epsilon_{2}}{r}\right]_{+} \\
& -\left[\frac{\boldsymbol{\alpha}_{1} \cdot \mathbf{p}_{1}}{4 m_{1} c}, \frac{\epsilon_{1} \epsilon_{2}}{2 r}\left(\delta^{i j}+\frac{r^{i} r^{j}}{r^{2}}\right) \alpha_{1}^{i} \alpha_{2}^{j}\right]_{+} \\
& +\left[\frac{\left(\boldsymbol{\alpha}_{1} \cdot \mathbf{p}_{1}\right) \mathbf{p}_{1}^{2}}{8 m_{1}^{3} c^{3}}, \frac{\epsilon_{1} \epsilon_{2}}{2 r}\left(\delta^{i j}+\frac{r^{i} r^{j}}{r^{2}}\right) \alpha_{1}^{i} \alpha_{2}^{j}\right]_{+}, \\
& (\mathcal{O O})=\left[\frac{\beta_{1} \mathbf{p}_{1}^{2}}{8 m_{1}^{2} c^{2}}+\frac{\beta_{2} \mathbf{p}_{2}^{2}}{8 m_{2}^{2} c^{2}}, \frac{\epsilon_{1} \epsilon_{2}}{2 r}\left(\delta^{i j}+\frac{r^{i} r^{j}}{r^{2}}\right) \alpha_{1}^{i} \alpha_{2}^{j}\right]_{+} .
\end{aligned}
$$

One can easily see that to carry out the transformation of the Salpeter equation in general, as a matter of fact, we ought to apply the prescription in a general form like (4), but extended to higher orders, as the odd-even and even-odd terms do not commute in the case under consideration. Still, if we supplement expression (6) with terms (4h, i), we will get a prescription for the transformation of the equation up to the fourth order in $1 / c$. It should be noted, however, that as the operator $(\mathcal{O O})$ is of the order $1 / c^{2}$, all the terms that contain this one in the thus obtained expression, except the ninth term from (6a), do not make any contribution to the desired accuracy, and hence, can be ignored (all of the terms with a difference of the masses in the denominators are among them).

Thus, after the evaluation, one obtains the transformation of the initial Hamiltonian (13) up to the order $1 / c^{4}$ in the following form: 


$$
\begin{aligned}
& H_{\mathrm{tr}}=\beta_{1} m_{1} c^{2}+\frac{\beta_{1} \mathbf{p}_{1}^{2}}{2 m_{1}}+\left(\beta_{1}+\beta_{2}\right) \frac{\epsilon_{1} \epsilon_{2}}{4 r}-\frac{\beta_{1}+\beta_{2}}{16 m_{1}^{2} c^{2}}\left[\left(\boldsymbol{\sigma}_{1} \cdot \mathbf{p}_{1}\right),\left[\left(\boldsymbol{\sigma}_{1} \cdot \mathbf{p}_{1}\right), \frac{\epsilon_{1} \epsilon_{2}}{r}\right]\right] \\
& -\frac{\beta_{1}+\beta_{2}}{16 m_{1} m_{2} c^{2}}\left[\left(\boldsymbol{\sigma}_{1} \cdot \mathbf{p}_{1}\right),\left[\left(\boldsymbol{\sigma}_{2} \cdot \mathbf{p}_{2}\right), \frac{\epsilon_{1} \epsilon_{2}}{2 r}\left(\delta^{i j}+\frac{r^{i} r^{j}}{r^{2}}\right) \sigma_{1}^{i} \sigma_{2}^{j}\right]_{+}\right]_{+}-\frac{\beta_{1} \mathbf{p}_{1}^{4}}{8 m_{1}^{3} c^{2}} \\
& -\frac{\beta_{1}}{32 m_{1}^{3} c^{4}}\left[\left(\boldsymbol{\sigma}_{1} \cdot \mathbf{p}_{1}\right), \frac{\epsilon_{1} \epsilon_{2}}{r}\right]^{2}+\frac{\beta_{1}}{32 m_{1} m_{2}^{2} c^{4}}\left[\left(\boldsymbol{\sigma}_{2} \cdot \mathbf{p}_{2}\right), \frac{\epsilon_{1} \epsilon_{2}}{2 r}\left(\delta^{i j}+\frac{r^{i} r^{j}}{r^{2}}\right) \sigma_{1}^{i} \sigma_{2}^{j}\right]_{+}^{2} \\
& +\frac{\beta_{1}}{32 m_{1} m_{2}^{2} c^{4}}\left[\left[\left(\boldsymbol{\sigma}_{1} \cdot \mathbf{p}_{1}\right), \frac{\epsilon_{1} \epsilon_{2}}{2 r}\left(\delta^{i j}+\frac{r^{i} r^{j}}{r^{2}}\right) \sigma_{1}^{i} \sigma_{2}^{j}\right]_{+},\left[\left(\boldsymbol{\sigma}_{2} \cdot \mathbf{p}_{2}\right), \frac{\epsilon_{1} \epsilon_{2}}{r}\right]\right] \\
& +\frac{\beta_{1}+\beta_{2}}{768 m_{1}^{4} c^{4}}\left\{\left[\left(\boldsymbol{\sigma}_{1} \cdot \mathbf{p}_{1}\right),\left[\left(\boldsymbol{\sigma}_{1} \cdot \mathbf{p}_{1}\right),\left[\left(\boldsymbol{\sigma}_{1} \cdot \mathbf{p}_{1}\right),\left[\left(\boldsymbol{\sigma}_{1} \cdot \mathbf{p}_{1}\right), \frac{\epsilon_{1} \epsilon_{2}}{r}\right]\right]\right]\right]\right. \\
& \left.+32\left[\left(\boldsymbol{\sigma}_{1} \cdot \mathbf{p}_{1}\right),\left[\left(\boldsymbol{\sigma}_{1} \cdot \mathbf{p}_{1}\right) \mathbf{p}_{1}^{2}, \frac{\epsilon_{1} \epsilon_{2}}{r}\right]\right]\right\} \\
& +\frac{\beta_{1}+\beta_{2}}{256 m_{1}^{2} m_{2}^{2} c^{4}}\left[\left(\boldsymbol{\sigma}_{1} \cdot \mathbf{p}_{1}\right),\left[\left(\boldsymbol{\sigma}_{1} \cdot \mathbf{p}_{1}\right),\left[\left(\boldsymbol{\sigma}_{2} \cdot \mathbf{p}_{2}\right),\left[\left(\boldsymbol{\sigma}_{2} \cdot \mathbf{p}_{2}\right), \frac{\epsilon_{1} \epsilon_{2}}{r}\right]\right]\right]\right] \\
& +\frac{\beta_{1}+\beta_{2}}{192 m_{1} m_{2}^{3} c^{4}}\left\{\left[\left(\boldsymbol{\sigma}_{1} \cdot \mathbf{p}_{1}\right),\left[\left(\boldsymbol{\sigma}_{2} \cdot \mathbf{p}_{2}\right),\left[\left(\boldsymbol{\sigma}_{2} \cdot \mathbf{p}_{2}\right),\left[\left(\boldsymbol{\sigma}_{2} \cdot \mathbf{p}_{2}\right), \frac{\epsilon_{1} \epsilon_{2}}{2 r}\left(\delta^{i j}+\frac{r^{i} r^{j}}{r^{2}}\right) \sigma_{1}^{i} \sigma_{2}^{j}\right]_{+}\right]_{+}\right]_{+}\right]_{+}\right. \\
& \left.+8\left[\left(\boldsymbol{\sigma}_{1} \cdot \mathbf{p}_{1}\right),\left[\left(\boldsymbol{\sigma}_{2} \cdot \mathbf{p}_{2}\right) \mathbf{p}_{2}^{2}, \frac{\epsilon_{1} \epsilon_{2}}{2 r}\left(\delta^{i j}+\frac{r^{i} r^{j}}{r^{2}}\right) \sigma_{1}^{i} \sigma_{2}^{j}\right]_{+}\right]_{+}\right\} \\
& -\frac{1}{64 m_{1}^{3} c^{4}}\left[\left[\mathbf{p}_{1}^{2}, \frac{\epsilon_{1} \epsilon_{2}}{r}\right], \frac{\beta_{1} \mathbf{p}_{1}^{2}}{m_{1}}+\frac{\beta_{2} \mathbf{p}_{2}^{2}}{m_{2}}+\left(\beta_{1}+\beta_{2}\right) \frac{\epsilon_{1} \epsilon_{2}}{r}\right] \\
& +\frac{1}{64 m_{1} m_{2}^{2} c^{4}}\left[\left[\left(\boldsymbol{\sigma}_{1} \cdot \mathbf{p}_{1}\right),\left[\left(\boldsymbol{\sigma}_{2} \cdot \mathbf{p}_{2}\right), \frac{\epsilon_{1} \epsilon_{2}}{2 r}\left(\delta^{i j}+\frac{r^{i} r^{j}}{r^{2}}\right) \sigma_{1}^{i} \sigma_{2}^{j}\right]\right]_{+}, \frac{\beta_{1} \mathbf{p}_{1}^{2}}{m_{1}}+\frac{\beta_{2} \mathbf{p}_{2}^{2}}{m_{2}}+\left(\beta_{1}+\beta_{2}\right) \frac{\epsilon_{1} \epsilon_{2}}{r}\right] \\
& +\frac{\beta_{1} \mathbf{p}_{1}^{6}}{16 m_{1}^{5} c^{4}}+\text { symm. terms. }
\end{aligned}
$$

Although this formula is only an intermediate result, the Hamiltonian written in such a form is quite transparent and suitable for a qualitative analysis. In addition, it is represented in the form similar to the one in (6) rewritten with due regard for the following notations:

$$
\begin{aligned}
& (\mathcal{E} \mathcal{E})=\frac{\epsilon_{1} \epsilon_{2}}{r}, \quad(\mathcal{O E})=c \boldsymbol{\alpha}_{1} \cdot \mathbf{p}_{1}, \quad(\mathcal{E O})=c \boldsymbol{\alpha}_{2} \cdot \mathbf{p}_{2} \\
& (\mathcal{O O})=-\frac{\epsilon_{1} \epsilon_{2}}{2 r}\left(\boldsymbol{\alpha}_{1} \cdot \boldsymbol{\alpha}_{2}+\frac{\left(\boldsymbol{\alpha}_{1} \cdot \mathbf{r}\right)\left(\boldsymbol{\alpha}_{2} \cdot \mathbf{r}\right)}{r^{2}}\right)
\end{aligned}
$$

therefore, is convenient to compare with the transformed Hamiltonian of the corresponding Breit equation [27].

It is well known that, in the Pauli approximation, the Breit equation and the Hermitian part of the Salpeter equation differ from one another by the $e^{4}$ terms coming from (4f), and which are responsible for the disagreement between the predicted and experimental values for the fine structure of helium. Obviously, the difference between the approximate forms for these equations will only increase in higher orders. Still, there are also many similarities between the $1 / c^{4}$-order parts of their transformed Hamiltonians. Thus the terms in (15), except (15d), are similar to the ones entering the transformed
Hamiltonian of the Breit equation. The $e^{4}$ terms, which we put together in (15b) and which are of the order $1 / \mathrm{m}^{3}$, do not disappear when one particle is in a positive energy state and the other is in a negative energy state. The similar terms, together with their symmetric ones, also occur among the terms coming from $(6 \mathrm{~b}, \mathrm{c}, \mathrm{e})$ rewritten with the use of the notations of (16). Note that all of them agree with those $e^{4}$ terms in the expansion of the Breit equation, which do not have the mass difference in the denominators, however, they are four times higher. Meanwhile, the $e^{2}$ terms (15c), which are of the order $1 / m^{4}$, exhibit another behavior. They have the factor $\beta_{1}+\beta_{2}$, and thus, become zero, if one particle is in a positive energy state and the other one is in a negative energy state. When both particles are in positive energy states, these terms coincide with the ones from $(6 \mathrm{~g}, \mathrm{~h}, \mathrm{i})$ rewritten for the case of the Breit equation, but all of them are opposite in sign to those provided that the two particles are in negative energy states. What concerns the terms (15d), there are no similar ones in the expansion of the Breit equation. One should remark that this part includes $e^{4}$ terms having the factor $\beta_{1}+\beta_{2}$ (the $e^{4}$ terms of (15b) are without this factor) and $e^{2}$ terms which do not disappear when the particles are in different energy states, as opposed to the terms (15c). 
It is easy to check, however, that these terms can be removed by an additional unitary transformation like that considered in the previous section. Namely, applying procedure (2) with generator

$$
\begin{aligned}
S & =-\frac{i}{32 c^{4}}\left[\frac{\mathbf{p}_{1}^{2}}{m_{1}^{3}}+\frac{\mathbf{p}_{2}^{2}}{m_{2}^{3}}, \frac{\epsilon_{1} \epsilon_{2}}{r}\right]+\frac{i}{32 m_{1} m_{2}^{2} c^{4}}\left[\left(\boldsymbol{\sigma}_{1} \cdot \mathbf{p}_{1}\right),\left[\left(\boldsymbol{\sigma}_{2} \cdot \mathbf{p}_{2}\right), \frac{\epsilon_{1} \epsilon_{2}}{2 r}\left(\delta^{i j}+\frac{r^{i} r^{j}}{r^{2}}\right) \sigma_{1}^{i} \sigma_{2}^{j}\right]\right]_{+} \\
& +\frac{i}{32 m_{1}^{2} m_{2} c^{4}}\left[\left(\boldsymbol{\sigma}_{1} \cdot \mathbf{p}_{1}\right),\left[\left(\boldsymbol{\sigma}_{2} \cdot \mathbf{p}_{2}\right), \frac{\epsilon_{1} \epsilon_{2}}{2 r}\left(\delta^{i j}+\frac{r^{i} r^{j}}{r^{2}}\right) \sigma_{1}^{i} \sigma_{2}^{j}\right]_{+}\right],
\end{aligned}
$$

to Hamiltonian (15), one eliminates the terms (15d) together with their symmetrical terms. This transformation only destroys these terms, without changing the rest part of the approximate Hamiltonian and without producing any new terms to the desired accuracy. Thus, with neglect of the terms $(15 \mathrm{~d}), H_{\mathrm{tr}}$, in the case under consideration, contains only the terms similar in their structure to those that occur in the transformed Hamiltonian for the Breit equation.

After calculating, we can thus represent the final form of the transformed Hamiltonian from expression (13), accurate to the order $1 / c^{4}$, as

$$
\begin{aligned}
H_{\mathrm{tr}} & =\beta_{1} m_{1} c^{2}+\frac{\beta_{1} \mathbf{p}_{1}^{2}}{2 m_{1}}+\left(\beta_{1}+\beta_{2}\right) \frac{\epsilon_{1} \epsilon_{2}}{4 r}-\left(\beta_{1}+\beta_{2}\right) \frac{\epsilon_{1} \epsilon_{2}}{8 m_{1}^{2} c^{2}}\left(2 \pi \delta(\mathbf{r})+\frac{\left(\mathbf{r} \times \mathbf{p}_{1}\right) \cdot \boldsymbol{\sigma}_{1}}{r^{3}}\right)-\left(\beta_{1}+\beta_{2}\right) \frac{\epsilon_{1} \epsilon_{2}}{16 m_{1} m_{2} c^{2}} \\
& \times\left\{\frac{2}{r}\left(\delta^{i j}+\frac{r^{i} r^{j}}{r^{2}}\right) p_{1}^{i} p_{2}^{j}+\frac{4}{r^{3}}\left(\mathbf{r} \times \mathbf{p}_{1}\right) \cdot \boldsymbol{\sigma}_{2}-\frac{1}{r^{3}}\left(\delta^{i j}-3 \frac{r^{i} r^{j}}{r^{2}}\right) \sigma_{1}^{i} \sigma_{2}^{j}+\frac{8 \pi}{3} \delta(\mathbf{r}) \boldsymbol{\sigma}_{1} \cdot \boldsymbol{\sigma}_{2}\right\}-\frac{\beta_{1} \mathbf{p}_{1}^{4}}{8 m_{1}^{3} c^{2}} \\
& +\frac{\beta_{1}\left(\epsilon_{1} \epsilon_{2}\right)^{2}}{32 m_{1}^{3} c^{4} r^{4}}+\frac{\beta_{1}\left(\epsilon_{1} \epsilon_{2}\right)^{2}}{16 m_{1} m_{2}^{2} c^{4} r}\left\{\frac{2}{r^{3}}-\frac{8 \pi}{3} \delta(\mathbf{r})+\frac{1}{r^{3}}\left(\left(\mathbf{r} \times \mathbf{p}_{2}\right) \cdot \boldsymbol{\sigma}_{2}+\frac{3}{2}\left(\mathbf{r} \times \mathbf{p}_{2}\right) \cdot \boldsymbol{\sigma}_{1}-\left(\mathbf{r} \times \mathbf{p}_{1}\right) \cdot \boldsymbol{\sigma}_{2}\right)\right. \\
& \left.-\frac{11}{r^{3}} \boldsymbol{\sigma}_{1} \cdot \boldsymbol{\sigma}_{2}+\left(\frac{9}{2 r^{3}}-\frac{2 \pi}{3} \delta(\mathbf{r})\right)\left(3 \delta^{i j}-\frac{r^{i} r^{j}}{r^{2}}\right) \sigma_{1}^{i} \sigma_{2}^{j}+\frac{1}{2 r}\left(\delta^{i j}+3 \frac{r^{i} r^{j}}{r^{2}}\right) p_{2}^{i} p_{2}^{j}-\frac{i}{r^{3}} \mathbf{r} \cdot \mathbf{p}_{2}\right\} \\
& +\left(\beta_{1}+\beta_{2}\right) \frac{\epsilon_{1} \epsilon_{2}}{256 m_{1}^{4} c^{4}}\left\{6\left[\mathbf{p}_{1}^{2}, 4 \pi \delta(\mathbf{r})+\frac{2}{r^{3}}\left(\mathbf{r} \times \mathbf{p}_{1}\right) \cdot \boldsymbol{\sigma}_{1}\right]+5\left[\mathbf{p}_{1}^{2},\left[\mathbf{p}_{1}^{2}, \frac{1}{r}\right]\right]\right\}+\left(\beta_{1}+\beta_{2}\right) \frac{\epsilon_{1} \epsilon_{2}}{256 m_{1}^{2} m_{2}^{2} c^{4}} \\
& \times\left\{-4 \pi \Delta \delta(\mathbf{r})-8 \pi\left(\nabla \delta(\mathbf{r}) \times \mathbf{p}_{1}\right) \cdot \boldsymbol{\sigma}_{1}+8 \pi\left(\nabla \delta(\mathbf{r}) \times \mathbf{p}_{2}\right) \cdot \boldsymbol{\sigma}_{2}+4\left[\frac{4 \pi}{3} \delta(\mathbf{r}) \delta^{i j}+\frac{1}{r^{3}}\left(\delta^{i j}-3 \frac{r^{i} r^{j}}{r^{2}}\right)\right]\right. \\
& \left.\times\left(\boldsymbol{\sigma}_{1} \times \mathbf{p}_{1}\right)^{i}\left(\boldsymbol{\sigma}_{2} \times \mathbf{p}_{2}\right)^{j}\right\}+\frac{\beta_{1}+\beta_{2}}{64 m_{1} m_{2}^{3} c^{4}}\left(2 p_{1}^{i}+\left(\boldsymbol{\sigma}_{1} \times \nabla\right)^{i}\right)\left\{\left(2 p_{2}^{j}-\left(\boldsymbol{\sigma}_{2} \times \nabla\right)^{j}\right)\left[\mathbf{p}_{2}^{2}, \frac{\epsilon_{1} \epsilon_{2}}{r}\left(\delta^{i j}+\frac{r^{i} r^{j}}{r^{2}}\right)\right]+\right. \\
& \left.+\left(\boldsymbol{\sigma}_{2} \times\left(2 i \mathbf{p}_{2}+\nabla\right)\right)^{j}\left[\mathbf{p}_{2}^{2}, \frac{\epsilon_{1} \epsilon_{2}}{2 r}\left(\delta^{i j}+\frac{r^{i} r^{j}}{r^{2}}\right)\right]\right\} \\
& +\frac{\beta_{1} \mathbf{p}_{1}^{6}}{16 m_{1}^{5} c^{4}}+\operatorname{symm}^{2} \operatorname{terms.}
\end{aligned}
$$

Here the terms to the second order are put together in (18a), and the others form the $1 / c^{4}$-order part of the transformed Hamiltonian.

The Chraplyvy transformation applied to Hamiltonian (13) gives its semirelativistic approximation involving terms (15d), which can be referred to extra terms, and which there is no need to include in the final equation in $H_{\mathrm{tr}}$. Yet, one cannot claim that they also arise in the $1 / c$ expansion obtained by a different method, e.g., by the method of expressing the small components of the spinor of the wave equation through its large components.

\section{SUMMARY}

In the present paper we have dealt with the two-body reduction method of Chraplyvy, and by its means we have briefly considered the problem of the expansion of equal-time relativistic two-body wave equations to higher orders in $1 / c$. For the case of two particles with unequal masses, we have found the fourth-order part of the transformed Hamiltonian in a general form expressed in terms of the initial even-even, even-odd, odd-even, and odd-odd operators. It turned out that in contrast with the case of the transformation to the second order, the final result of the transformation to higher orders depended on the order of application of the generators, i.e., as these functions do not commute with each other in a general case, certain extra terms can appear in the thus obtained $1 / c$ expansion of the equation. Still, as we have shown, the two transformed Hamiltonians, each correct to $1 / c^{4}$, can be converted one into the other by an additional unitary transformation, so that terms of this kind are eliminated, and without changing the remaining part of the Hamiltonians as well. The occurrence of the extra terms in $H_{\mathrm{tr}}$ may be a feature of the expansion of 


\section{ALEXEI TUROVSKY}

relativistic equations to higher orders, within the frame of the method under consideration at least. Thus, the higher-order transformed Hamiltonian is defined up to a unitary transformation with an even-even generating function of the order $1 / c^{4}$. This is in agreement with the conclusions made by Pursey in [16], where ambiguities in the method were discussed.

For illustration, we have considered the $1 / c$ expansion of the Salpeter equation with the Breit interaction up to the terms of the fourth order. Due to the Casimir projection operators, the $1 / c^{4}$-order part of the transformed Hamiltonian obtained from equation (13) is much simpler than the analogous part in the transformation of the corresponding Breit equation, and involves no terms with mass differences in the denominators or terms similar to those of (6d) by their structure. However, none of the obtained $e^{4}$ terms in the expansion, except the extra terms, disappears when one particle is in a positive energy state and the other is in a negative energy state, meanwhile all the $e^{2}$ terms become zero. With that all the interaction terms to the order $1 / c^{2}$ in the transformed Hamiltonian derived from the equation under consideration have the factor $\beta_{1}+\beta_{2}$, but all of them are $e^{2}$ terms and vanish provided one particle is in a positive energy state and the other is in a negative energy state (see also [15]). This may indicate that the $e^{4}$ terms in the obtained expansion are nonphysical ones similarly to the nonphysical $e^{4}$ terms of $1 / c^{2}$ coming from (4f) in the case of the corresponding Breit equation. Notice that a number of the $1 / c^{4}$-order terms obtained here agree up to a numerical multiplier, and in many features, are similar to the relativistic corrections in the $\alpha^{6} m c^{2}$ Hamiltonian for an arbitrary light atom [22]. We should note, however, that here we have dealt with the initial equation represented in the Hermitian form, which in general differs from the original Salpeter equation (see, e.g., [32] for details).

In fact, the approach applied here to expand two-body equations is a straightforward way of deriving many $1 / c^{4}$ order terms giving $\alpha^{6} m c^{2}$ corrections to the energy levels for a hydrogen-like atom. We emphasize, however, that this work has been devoted mainly to the generalization of the FW canonical transformation to the two-particle problem. Although we have illustrated the application of the results on the example of a relativistic equation, the application to a real atomic system and calculation of the energy eigenvalues are beyond the purpose of the paper. Because of the simple form of the initial interaction we used in the equation, the obtained approximate Hamiltonian is not complete and does not take into consideration many QED effects. The derivation of the total effective Hamiltonian contributing to $\alpha^{6} m c^{2}$ to the energy levels of a hydrogen-like atom by means of the method treated here requires the use of quantum field theories and the diagram technique as well, is of interest in itself, and could be the subject of future investigations.

\section{ACKNOWLEDGMENTS}

I wish to thank all my colleagues at my department for interesting and useful discussions, and I am also grateful to Professor I. V. Simenog for his critical comments.
[1] T. Tanaka, A. Suzuki, M. Kimura, Z. Phys. A 353, 79 (1995).

[2] A. G. Nikitin, V. V. Tretynyk, Int. J. Mod. Phys. 12, 4369 (1997).

[3] N. Kasari, Y. Yamaguchi, Phys. Lett. B 508, 198 (2001).

[4] I. V. Simenog, A. I. Turovsky, Ukr. Fiz. Zh. 46, 391 (2001); J. Phys. Stud. 8, 23 (2004).

[5] H. W. Crater, P. Van Alstine, Phys. Rev. D 70, 034026 (2004).

[6] S. S. Pikh, J. Phys. Stud. 16, 4101 (2012).

[7] H. A. Bethe, E. E. Salpeter, Quantum Mechanics of Oneand Two-Electron Atoms (Plenum, New York, 1977).

[8] A. E. S. Green, T. Sawada, Rev. Mod. Phys. 39, 594 (1967).

[9] W. Lucha, F. F. Schöberl, D. Gromes, Phys. Rep. 200, 127 (1991).

[10] L. L. Foldy, S. A. Wouthuysen, Phys. Rev. 78, 29 (1950); J. D. Bjorken, S. D. Drell, Relativistic Quantum Mechanics (McGraw-Hill, New York, 1964).

[11] Z. V. Chraplyvy, Phys. Rev. 91, 388 (1953).

[12] Z. V. Chraplyvy, Phys. Rev. 92, 1310 (1953).

[13] M. L. Lewis, V. W. Hughes, Phys. Rev. A 8, 625 (1973).

[14] E. E. Salpeter, Phys. Rev. 87, 328 (1952).

[15] W. A. Barker, F. N. Glover, Phys. Rev. 99, 317 (1955).

[16] D. L. Pursey, Nucl. Phys. 8, 595 (1958).

[17] E. de Vries, J. E. Jonker, Nucl. Phys. B 6, 213 (1968).
[18] H. Janyszek, Acta Phys. Pol. B5, 447 (1974); Acta Phys. Pol. B5, 583 (1974).

[19] C. E. Aguiar, A. N. F. Aleixo, C. A. Bertulani, Phys. Rev. C 42, 2180 (1990).

[20] M. Douglas, N. M. Kroll, Ann. Phys. (N.Y) 82, 89 (1974).

[21] J. W. Darewych, A. Duviryak, Phys. Rev. A 66, 032102 (2002).

[22] K. Pachucki, Phys. Rev. A 71, 012503 (2005).

[23] K. Pachucki, V. A. Yerokhin, Phys. Rev. Lett. 104, 070403 (2010).

[24] A. P. Martynenko, Yad. Fiz. 71, 126 (2008) [Phys. At. Nucl. 71, 125 (2008)]; N. A. Boikova, Yu. N. Tyukhtyaev, R. N. Faustov, Yad. Fiz. 74, 68 (2011) [Phys. At. Nucl. 74, 67 (2011)].

[25] E. N. Elekina, A. A. Krutov, A. P. Martynenko, Pis'ma Fiz. Elem. Chast. At. Yadra 8, 554 (2011) [Part. Nucl. Lett. 8, 331 (2011)].

[26] S. G. Karshenboim, V. G. Ivanov, E. Yu. Korzinin, Phys. Rev. A 85, 032509 (2012).

[27] A. I. Turovsky, Ukr. J. Phys. 56, 5 (2011).

[28] N. Brambilla, D. Eiras, A. Pineda, J. Soto, A. Vairo, Phys. Rev. D 67, 034018 (2003).

[29] P. Indelicato, O. Gorceix, J. P. Desclaux, J. Phys. B 20, 651 (1987); P. Indelicato, J. P. Desclaux, Phys. Rev. A 42, 5139 (1990). 
[30] A. I. Akhiezer, V. B. Berestetskii, Quantum Electrodynamics (Wiley, New York, 1965).

[31] V. B. Berestetskii, E. M. Lifshitz, L. P. Pitaevskii, Quan- tum Electrodynamics (Pergamon Press, Oxford, 1982).

[32] G. Feldman, T. Fulton, J. Townsend, Phys. Rev. A 8, 1149 (1973).

\title{
ПЕРЕТВОРЕННЯ ХРАПЛИВОГО ТА ДЕЯКІ ОСОБЛИВОСТІ ЙОГО ЗАСТОСУВАННЯ ДЛЯ РОЗКЛАДІВ ДО ВИЩИХ ПОРЯДКІВ
}

\author{
Олексій Туровський \\ Інститут теоретичної фізики ім. М. М. Боголюбова НАН Украӥни, \\ вул. Метрологічна, 14б, Київ, 03680, Украӥна
}

\begin{abstract}
На базі узагальнення Храпливого методу Фолді-Вутгайзена до двочастинкового випадку розглянуто проблему розкладу релятивістських рівнянь для двох частинок за степенями $1 / c$ до вищих порядків. Для частинок із різними масами отримано трансформований гамільтоніан до порядку $1 / c^{4}$. Установлено, що залежно від порядку застосування генеруючих функцій для першої ітерації частину трансформованого гамільтоніана, яка містить члени вищого порядку, можна отримати у двох різних формах, які, однак, перетворюються одна в одну за допомогою додаткового унітарного перетворення. Обговорено подібні неоднозначності в такій процедурі. Як приклад для ілюстрації взято рівняння Солпітера в ермітовій формі із взаємодією Брейта та, використовуючи метод, що розглядається, виконано його редукцію до наближеного рівняння, яке включає всі члени порядку $1 / c^{4}$. Отримані результати можна застосувати для нерелятивістського розкладу двочастинкових хвильових рівнянь із різноманітними потенціалами взаємодії, для вивчення тонкої та надтонкої структури рівнів воднеподібних атомів. Вони можуть становити загальний теоретичний
\end{abstract} інтерес. 